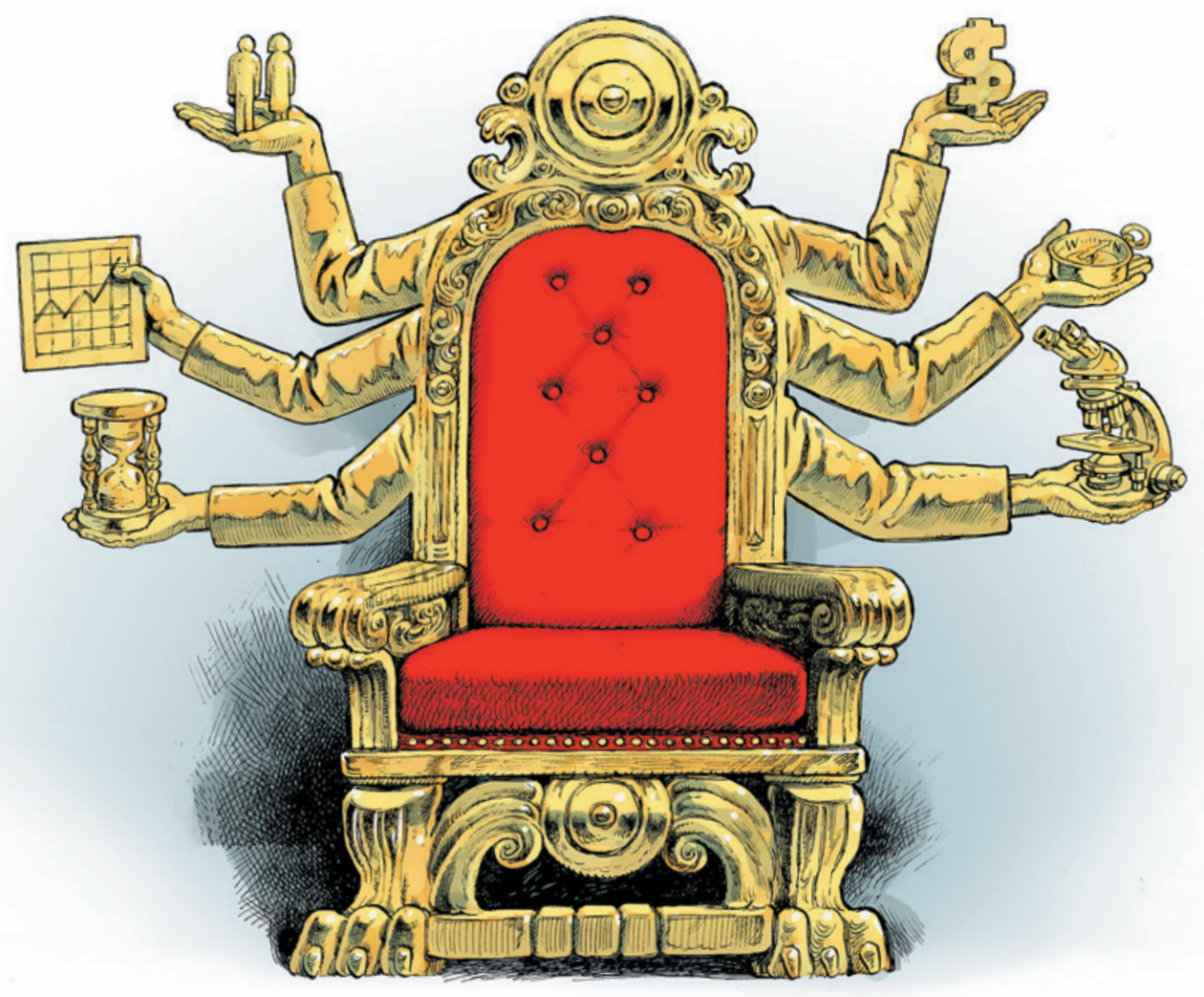

\title{
Ten tips for choosing an academic chair
}

\section{Agree what is needed, look for leaders with fire in their belly and emotional intelligence, and support them, advise Pierre-Alain Clavien and Joseph Deiss.}

$\mathrm{T}$ The academic world has changed greatly in recent decades, so demands on its leaders have too. Departmental chairs, deans, facility directors and other leaders are now expected to power research, attract funding, manage investments, engage with policymakers, woo the media and train personnel.

Finding people who can manage these demands simultaneously is difficult. Botched appointments are costly - intellectually, emotionally and financially - for universities, students, research and sometimes for hospitals and patients too. Surprisingly, there is little data on the selection processes of academic chairs ${ }^{1,2}$.

Here, we relate a recent exercise in selecting a chair for a position in clinical academic medicine that in our view holds lessons for the appointment of science leaders more generally. Through a formal consensus process involving leaders from industry, policy and academia, we have distilled a set of principles - telegraphed here (see 'Checklist for high-level hiring') - for making highlevel hires. Although many seem unsurprising, they are too often ignored.

\section{HEROIC EFFORT}

Departmental chairs of clinical academic medicine were traditionally referred to as heroic 'tri-legs' - leaders across three realms: patient care, research and the teaching of vast disciplines such as surgery. However, the required skill set has grown, with the emergence of many sub-specialities, new financial and legal constraints, and the changing demands of students and patients.

There have been notorious failures to appoint appropriate chairs. Too often, hiring committees plump for top-notch researchers who lack social skills or they go for socially adroit individuals with poor academic credibility. Unsuitable choices are usually made as a result of a selection process driven by bias, self-interest and power games by members of the search committee, which often leads to the exclusion of the best candidates from the outset. But the problems go wider and deeper.

In search of a more evidence-based way to pinpoint the best people for the top jobs, an international group of experts was convened by the University of Zurich and University Hospital Zurich, along with other Swiss universities. Using an approach known as the Zurich-Danish model of consensus conferences $^{3}$, an independent, unbiased 12-person jury comprising specialists of several kinds - from science to business - finalized recommendations prepared by nine panels of experts (one of us, P.-A.C., served as president of the local organizing committee). This format was originally developed to assess controversial treatments of diseases such as liver cancer ${ }^{4}$ or neuroendocrine tumours ${ }^{5}$ or to guide the development of new medical 


\section{Checklist for high-level hiring}

\begin{abstract}
Align needs of all areas of responsibility. One of the main causes of hiring failures is conflict between the expectations of the institutions involved in the selection process - the hospital and the university. For example, hospital chief executives and boards may look for cost-effective or low-cost medicine, and welcome an obedient manager with modest expectations. By contrast, an ambitious university or medical school may welcome original thinkers with initiative, attracting atypical researchers and grants to challenge paradigms. Such institutions must discuss and agree their expectations.
\end{abstract}

\section{Screen search-committee members for conflicts of interest. This is the most common source of bias. It may lead to the exclusion of highly qualified individuals on the basis of subjective and misleading criticisms. Any negative statements about a candidate should be made in writing to secure a measure of accountability.}

Articulate requirements clearly in the job advertisement. Before candidates can be considered, the hiring committee must clearly state the standards required in clinical ability, research and teaching.
Articulate the offer clearly. Early in the hiring process, assess whether the resources of the institution and local conditions meet the ambition, motivation and other expectations of a potential candidate.

\section{Ensure accountability in the selection} process. The US academic system usually gives full responsibility for the final choice to the dean of an institution. In most other parts of the world, it should be made clearer who the decision-makers are.

Publications and citations are necessary but not sufficient. Bibliometrics must be interpreted with expertise - they can be gamed $^{7}$. Measure research qualitatively as well as quantitatively ${ }^{8}$. A portfolio of scientific activities should also be required, showcasing the quality and continuity of a candidate's research. Evidence of networking activities and successful pupils should also be sought.

Don't expect MBAs. Many academic centres hope for recruits with a deep knowledge of management and an ability to control tight budgets, as well as requiring an outstanding researcher, clinician and educator. They are hunting unicorns. More realistic is a chair with clinical, academic and social credentials, who can delegate work to business managers with complementary skills.

Seek strong emotional, personal and social skills. Leaders need to be highly intelligent in communication and relationship-building to support and motivate interdisciplinary teams, convey integrity, adapt to change ${ }^{9}$ and to empathize with patients. This feature cannot be compensated for by other qualities. People succeed when they treat the individuals around them well.

Find someone with fire in their belly and stoke it. Chairs need to be ready to fight for their academic mission and to identify strategies to minimize the administrative burden imposed on them and their academic colleagues. The passion of a new chair should be maintained by academic freedom, good infrastructure and room for development. These factors are much more important than salary benefits in attracting - and keeping highly qualified individuals.

Support your hire. Even the best people need regular feedback, mentoring and development. Set up a process to do so. technologies and techniques, including laparoscopic liver surgery ${ }^{6}$.

Over two-and-a-half days, panels of three to six participants addressed questions such as how to attract the best candidates, how to use scientometrics and how to assess personal and social skills. Panel members were deans, chief executives or professors from major academic centres around the world and included psychologists, epidemiologists, statisticians, economists and other specialists. The report of each panel is available at www.chair4medicine.uzh.ch.

The jurors ranged from the chief executive of a major insurance company and a Chemistry Nobel laureate to a student and a patient representative. One of us (J.D.), served as chair and Philip Campbell, editor-in-chief of this journal, was vice-chair of the jury. Following the conference, the jury agreed on 32 guidelines for standardized, yet versatile, selection criteria (see Supplementary Information for full list, go.nature.com/juzwjo).

One shortcoming of the process was the under-representation of women. The jury included three women and nine men. Less than $20 \%$ of panel members were female. This is a corollary of the gender imbalance in the pool from which we were recruiting: leaders inside and outside of academia. This is regrettable, especially for an exercise in a field such as medicine, which overall is more gender balanced than, say, particle physics. Analogous exercises in other disciplines should take greater pains to have more equality among participants.

\section{BEST PRACTICE}

These recommendations should be useful across broad areas of medicine, ranging from psychiatry to transplantation surgery. They may also support changes in countries with a tradition of inheritable leadership positions. Can a standardized selection process benefit different cultures with diverse health-care systems, those with high standards in medicine as well as resource-poor countries fighting for more vital issues? We believe so: leadership qualities have many common facets regardless of surroundings, and rapid globalization is flattening many cultural disparities.

Although many of our recommendations may seem obvious, we feel confident that the details - adjusted to local culture, norms and needs - will guide successful searches in medicine and beyond.

Pierre-Alain Clavien is professor and chairman of the department of surgery, University Hospital Zurich, Zurich,
Switzerland, and associate professor at the Paul Brousse Hospital, University of Paris Sud, Paris, France. Joseph Deiss is former president of Switzerland and of the United Nations General Assembly. e-mail:clavien@access.uzh.ch

1. Grigsby, R. K., Hefner, D. S., Souba, W. W. \& Kirch, D. G. Acad. Med. 79, 571-577 (2004).

2. Shumacker, H. B. Jr Ann. R. Coll. Surg. Eng. 65, 59-60 (1983).

3. Lesurtel, M., Perrier, A., Bossuyt, P. M., Langer, B. \& Clavien, P. A. Surgery 155, 390-397 (2014).

4. Clavien, P. A. et al. Lancet Oncol. 13, e11-e22 (2012).

5. Frilling, A. et al. Lancet Oncol. 15, e8-e21 (2014).

6. Wakabayashi, G. et al. Ann. Surg. 261, 619-629 (2015).

7. Kerr, N. L. Pers. Soc. Psychol. Rev. 2, 196-217 (1998).

8. Abbott, A. Nature 465, 860-862 (2010).

9. Shore, D. A. \& Kupferberg, E. D. J. Health Commun. 19, 275-281 (2014).

\section{CORRECTION}

The Comment 'Put people at the centre of global risk management' (J. W. Erisman et al. Nature 519, 151-153; 2015) gave an incorrect affiliation for co-author Philippe Ciais. He is a senior researcher at the Laboratory for Climate Sciences and the Environment, University of Versailles, France. 